Norois

Environnement, aménagement, société

$233 \mid 2014$

Mobilité, santé et développement territorial : de nouveaux défis pour la gouvernance des territoires ruraux

\title{
Vers une grille d'analyse de l'expérience géographique : cas-type dans la campagne au Québec
}

Towards an analytical framework of the geographical experience: a test case from the Quebec countryside

\section{Laurie Guimond}

\section{CpenEdition}

\section{Journals}

Édition électronique

URL : https://journals.openedition.org/norois/5253

DOI : $10.4000 /$ norois. 5253

ISBN : 978-2-7535-4127-6

ISSN : 1760-8546

Éditeur

Presses universitaires de Rennes

\section{Édition imprimée}

Date de publication : 20 décembre 2014

Pagination : 11-24

ISBN : 978-2-7535-4083-5

ISSN : 0029-182X

Référence électronique

Laurie Guimond, « Vers une grille d'analyse de l'expérience géographique : cas-type dans la campagne au Québec », Norois [En ligne], 233 | 2014, mis en ligne le 20 décembre 2016, consulté le 14 janvier 2022. URL : http://journals.openedition.org/norois/5253; DOI : https://doi.org/10.4000/norois.5253 


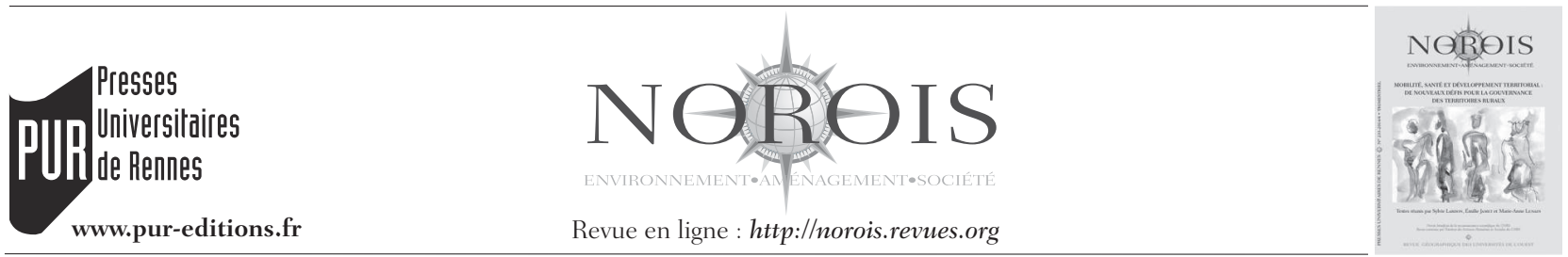

\title{
Vers une grille d'analyse de l'expérience géographique : cas-type dans la campagne au Québec
}

\author{
Towards an Analytical Framework of the Geographical Experience: \\ A Test Case from the Québec Countryside
}

\author{
Laurie Guimond
}

Département de géographie, Université du Québec à Montréal, Case postale 8888, Succursale Centre-Ville Montréal (Québec, Canada), H3C 3P8 (guimond.laurie@uqam.ca)

\begin{abstract}
Résumé : Cet article propose une grille d'analyse de l'expérience géographique pour étudier les relations que les individus entretiennent avec leur milieu de vie en se penchant plus spécifiquement sur celui de la campagne. Il suggère d'explorer les significations que les populations rurales y confèrent, en s'attachant aux modalités et finalités de leur appartenance, à leurs identités territoriales et à leurs représentations des lieux. Une attention particulière est portée à leurs origines géographiques et sociales ainsi qu’à leurs pratiques quotidiennes de mobilité, de sociabilité et d'engagement. En outre, l’approche préconisée insiste sur la nécessité de tenir compte des facteurs structuraux et du milieu modulant l'expérience géographique. Un cas-type retenu dans la campagne au Québec teste et met en relation ces diverses dimensions. Notre proposition semble fournir un cadre fécond pour la compréhension des différentes façons d'habiter, de cohabiter et d'expérimenter la campagne contemporaine.
\end{abstract}

\begin{abstract}
This article proposes an analytical framework of the geographical experience to study the interactions between individuals and the milieu they inhabit, in this case, the countryside. It suggests to explore the meanings that rural populations confer to the countryside, through their sense of belonging, their territorial identities, and their representations of the places that shape their everyday lives. A particular attention is paid to their geographical and social origins as well as their everyday practices of mobilily, sociability and involvement. Moreover, the advocated approach insists on the necessity to consider structural factors and the milieu which affect geographical experience. A test case from the Québec countryside illustrates the interactions between the proposed dimensions. Our analytical framework seems to enhance the understanding of the different ways of inhabiting, cohabiting and experiencing the contemporary countryside.
\end{abstract}

Mots clés : Expérience géographique - sens des lieux - populations rurales - campagne - Québec

Keywords: Geographical experience - sense of place - rural populations - countryside - Québec

\section{INTRODUCTION}

Marcher dans la forêt sur un lit d'épines de sapin, respirer l'air salin ou une odeur nauséabonde de fertilisant naturel, visiter des amis dans son village natal, ignorer son voisin car il tond sa pelouse le dimanche matin à sept heures, se souvenir de son enfance passée au bord du fleuve ou du lac, déneiger sa cour l'hiver, reconduire son adolescent dans le village voisin faute de transport en commun font partie de l'expérience de la campagne. L'expérience renvoie ainsi à tout un ensemble de pratiques, de représentations 
et d'émotions, qui caractérisent la vie quotidienne. Du point de vue des géographes, qui l'analysent depuis maintenant plus de 40 ans par le truchement de divers concepts comme ceux d'espace vécu, de «place » et de mode d'habiter, l'expérience ne peut être envisagée sans tenir compte des lieux concrets qui la structurent. L'expérience géographique relève des relations matérielles comme idéelles que les individus entretiennent avec les lieux qu'ils fréquentent au quotidien. Comment redonner sens à de telles relations avec la campagne d'aujourd'hui?

Le Québec accueille une population rurale composée de plus de 1,5 million d'individus qui occupent $90 \%$ du territoire habité et qui représentent près de $20 \%$ des Québécois (Statistique Canada, recensement de 2011). Avec une superficie supérieure à 1,3 million de kilomètres carrés ${ }^{2}$, le relief du Québec est jalonné par les montagnes appalachiennes, les basses terres du Saint-Laurent et, plus aux marges de l'écoumène, le Bouclier canadien. Ses vastes plans d'eau, dont le fleuve SaintLaurent, le caractérisent tout autant, à l'instar de ses saisons accusées et fortement marquées par la nordicité. Bien que l'économie rurale québécoise tend à se diversifier et à se tertiariser, la proportion des emplois dans les secteurs primaires (agriculture, foresterie, activités minières, pêcheries) et secondaires (secteurs manufacturier et de la construction) atteint un peu plus de $30 \%$ (ibid.). Comme ailleurs, les campagnes québécoises connaissent d'importantes mutations sur le plan des usages, des populations, des paysages, des modes d'habiter ou des modes de gouvernance (Domon et al., 2011; Jean et Lafontaine, 2010; Simard, 2011 ; Simard et al., n.p.). Une transformation qui retient particulièrement notre intérêt est celle de la recomposition sociodémographique par l'installation en permanence d'individus d'origine urbaine dans les campagnes. Ce phénomène touche de nombreuses municipalités rurales de régions administratives localisées tant à proximité des grandes villes du Québec (Laurentides, Chaudière-Appalaches, Outaouais...), que dans des régions intermédiaires (Centre-du-Québec, Mauricie...) ou éloignées (Gaspésie-Îles-de-la-Madeleine, Côte-Nord...). Cette recomposition invite à réfléchir les campagnes sur la base de l'expérience de ceux qui l'habitent.

À cet effet, les outils existants sont lacunaires. Diverses typologies, le plus souvent aux visées démographiques, statistiques et politico-administratives, sont mises en avant pour définir les campagnes québécoises. Quasi systématiquement, elles sont catégorisées en fonction de la distance à la ville et de ses effets économiques (Jean, 2006). C'est le cas de la typologie de Jean et ses collègues (2014), retenue dans la Politique nationale de la ruralité 2014-2024, à savoir : milieux ruraux périmétropolitains, milieux ruraux situés au voisinage d'une ville de moyenne importance, milieux essentiellement ruraux non éloignés des milieux densément peuplés, milieux ruraux éloignés du Québec densément peuplé. À cette fin fonctionnelle, le vocable « rural » est souvent juxtaposé aux notions d'espace, de territoire, de milieu et de région, en faisant fi de la signification de ces concepts géographiques fondamentaux ${ }^{1}$. Ces typologies ne reflètent qu'une infime partie de la réalité des campagnes québécoises qui sont, avant tout, un milieu de vie habité, pratiqué, socialisé, rêvé et renégocié au quotidien par des populations hétéroclites.

De ce fait, nous proposons plutôt une approche expérientielle de cette "ruralité », car au-delà des critères numériques, ce sont des individus qui habitent et expérimentent la campagne. À notre sens, la notion de « campagne » renvoie à cette forte signification expérientielle, voire culturelle, sociale et symbolique qui révèle un état d'esprit. Notre grille d'analyse propose une exploration des différentes façons de vivre la campagne et des significations que lui confèrent ses habitants. Cela, à l'image des travaux de Ruiz et Domon qui, par la notion de " communauté de relations au paysage », étudient les populations rurales sur la base de leur expérience avec le paysage (2013). En complément des recherches menées par des géographes, sociologues et ruralistes qui se sont attachés à scruter, souvent de façon isolée, certaines facettes de l'expérience de la ruralité, notre grille d'analyse met en dialogue différentes dimensions relatives à cette expérience.

Fondamentalement géographique, l'expérience de la campagne s'enracine dans des lieux, qui sont à la fois pratiqués, socialisés, vécus, rêvés et chargés

\footnotetext{
1. Par exemple, selon Statistique Canada, les « régions rurales " se situent à l'extérieur des «centres de population ». Ces dernières comprennent une concentration démographique d'au moins 1000 habitants et une densité de population d'au moins 400 habitants au kilomètre carré. Les centres de population sont classés selon la taille de leur population : petits ( 1000 à 29999 habitants); moyens (30 000 à 99999 habitants); grands (100000 habitants et plus) (recensement de 2011).
} 
de sens. Portée par des individus, elle s'appréhende d'abord par l'usage quotidien des lieux. Au-delà de leur caractère routinier et ordinaire, les pratiques quotidiennes dévoilent l'expérience plus large, nourrie par ces individus et leurs rapports subjectifs aux lieux, mais aussi influencée par le contexte dans lequel elles s'opèrent.

Notre approche a ceci d'intéressant qu'elle élargit de deux façons l'analyse des seuls pratiques et sens des lieux. Premièrement, elle leur adjoint le concept d'habitus qui offre la possibilité de mieux « contextualiser » l'expérience des lieux, en replaçant l'individu comme être social au cœur de celle-ci. Qui plus est, l'habitus éclaire cette expérience par le fait même qu'il rattache l'individu et les groupes aux conditions sociales qui déterminent leurs pratiques et leurs représentations, mais aussi leurs intérêts, leurs réseaux ainsi que leurs manières d'interagir et de se présenter. Deuxièmement, notre approche situe l'individu et son expérience dans le contexte plus large dans lequel il évolue. Cela implique que l'on considère non seulement son milieu de vie immédiat, c'est-à-dire son voisinage, sa localité, voire la région où il habite, dans ce qu'ils confèrent de spécifique à l'expérience, mais aussi l'ensemble des milieux façonnant l'expérience géographique, qui débordent ainsi sur les autres échelles du social. Cette approche s'intéresse aux facteurs structuraux qui en conditionnent l'évolution, parmi lesquels les transformations générales de l'économie, de la nature, de la société, du politique.

C'est par le recours au récit de vie spatialisé que nous testons cette grille d'analyse. Le cas-type retenu est issu d'une thèse de doctorat sur les migrations ville-campagne et l'espace de rencontre des populations rurales au Québec (Guimond, 2012). Les entrevues qualitatives approfondies ont permis de penser le sens des lieux et les pratiques avec une approche en termes d'habitus. Couplée à cette méthode, l'observation participante a permis de mieux contextualiser les propos des participants. Concrètement, la thèse démontre que l'étude de l'expérience géographique fait ressortir les façons polymorphes dont les populations rurales habitent, expérimentent et se représentent la campagne, ce qui est fait ici à partir d'un cas-type.

\section{Assises THÉORIQUES : ENTRE GÉOGRAPHICITÉ, MODES D'HABITER ET EXPÉRIENCE GÉOGRAPHIQUE}

La relation universelle entre l'homme et la Terre se matérialise par des pratiques et des valeurs qui forment son mode d'existence. La géographie d'Eric Dardel, qui, de façon précoce, a annoncé le tournant humaniste en géographie enseigne : «Amour du sol natal ou recherche du dépaysement, une relation concrète se noue entre l'homme et la Terre, une géographicité de l'homme comme mode de son existence et de son destin » (1952, 1 et 2). L'homme détient le moyen de donner un sens à ses actions, à cette relation dialectique existentielle qu'il entretient avec la Terre. La contribution de Dardel s'attache à " approfondir la relation fondatrice de l'homme à son milieu de vie », aux milieux qu'il habite (Bédard, 2011 , 282). Malgré cette riche contribution à tort reléguée aux oubliettes, ce ne sera que dans les années 1970 que la géographie se donnera pour mission d'élucider les rapports matériels comme idéels aux lieux, voire les rapports existentiels. Les travaux de la géographie humaniste anglosaxonne sur l'espace social, «place » et « sense of place » (Buttimer, 1969; Relph, 1976; Tuan, 1977) ainsi que française sur l'espace vécu (Frémont, 1974, 1976) sont déterminants à cet égard. Ils insistent tous sur le fait que l'expérience renvoie à un ensemble de sentiments, d'émotions et de rapports subjectifs aux lieux et aux autres qui viennent enrichir les connaissances de la vie quotidienne.

La compréhension géographique des rapports hommes-milieux fut aussi affinée par des réflexions sur les modes d'habiter en insistant sur les actions de « demeurer, travailler, circuler, vivre ensemble » (Mathieu, 2007). Pour la chercheure, ces rapports s'appuient sur les relations indissociables entre lieux et habitants. En fait, les habitants habitent et sont habités par une panoplie de lieux et, réciproquement, ils affectent ces lieux par leurs pratiques (2014). Au-delà du rapport à l'habitation (le logis), l'acte d'habiter passe par des pratiques sociales et spatiales à différentes échelles, voire des activités quotidiennes individuelles et collectives qui sont indéniablement nourries par les représentations et le sens des lieux. Les actions d'habiter, de se déplacer et de cohabiter constituent le socle de l'expé- 
rience géographique. D'où la richesse du concept de mode d'habiter qui intègre les façons de faire et de vivre l'expérience des lieux et qui articule «l'espace de vie, la mobilité, le sens des lieux et les relations sociales » (Schmitz, 2012). La notion d'expérience géographique affine la compréhension de ces pratiques et du sens des lieux en les contextualisant. Nous proposons de le faire de deux façons, d'abord en nous penchant sur le profil de l'individu qui l'expérimente, ensuite en nous attachant à l'ensemble des circonstances dans lesquelles s'inscrit son expérience géographique.

Alors que la géographie maitrise bien les pratiques et le sens des lieux dans l'analyse de l'expérience géographique, elle semble parfois négliger l'individu au cœur de celle-ci. Pourtant, comme le relève Di Méo : «L'homme qui se représente l'espace et son milieu, qui le façonne et le transforme est à la fois une conscience, une psyché, un sujet philosophique et politique, un individu, une personne, un être humain et un être social » (2000, 37). L'individu est avant tout socialisé et il a une expérience antérieure le rattachant à des groupes d'appartenance qui se déclinent selon leur classe sociale, leur origine géographique, leur sexe, leur personnalité, leurs comportements, leurs connaissances... Ses repères individuels font qu'il n'est pas libre d'appartenance. C'est surtout ce qui justifie notre recours à la notion d'expérience géographique qui tient compte à la fois de l'expérience individuelle et du profil de celui qui la produit. À cela s'ajoutent deux conditions extérieures étroitement liées qui structurent l'expérience géographique, soit le milieu et les facteurs structuraux.

L'expérience géographique des individus et des groupes auxquels ils appartiennent s'opère dans un milieu avec ses propres conditions d'existence : localisation et caractéristiques naturelles, paysagères, physiques et biogéographiques, situation sociodémographique, projets politiques, contexte économique, encadrement institutionnel, histoire... De ce fait, chaque milieu ne se développe pas au même rythme, ni avec la même intensité, ni de la même façon. Le milieu structure l'expérience géographique et occupe un rôle déterminant tant par la nature des liens sociaux entre les individus, que par leurs conceptions et leurs façons de voir l'Autre. Le milieu peut se décliner à différentes échelles, de la localité à la région, des milieux urbains, ruraux, littoraux, alpins, nordiques, à l'espace virtuel... Ce qui se produit à une échelle donnée agit sur ce qui se produit à une autre, en particulier dans le contexte actuel de mobilité (Stock, 2004). L'expérience des lieux est configurée aussi bien par les migrations prolongées que par la mobilité quotidienne, que ce soit de la maison au lieu de travail, d'un village à un autre, de la campagne à la ville, d'une part et d'autre de la frontière.

L'individu se situe dans un milieu de vie, mais aussi à une époque donnée, avec des conditions économiques, écologiques et démographiques, puis des influences politiques et sociétales notamment véhiculées dans la médiasphère. L'expérience géographique s'élabore dans un contexte plus large que le milieu, et qui relève de divers facteurs structuraux. Ces mécanismes sont parfois peu perceptibles, mais ils affectent assurément l'expérience géographique. D'où l'importance de s'intéresser aux structures et aux contraintes qui agissent sur la vie quotidienne (Highmore, 2002).

Pour déceler ces mécanismes et mieux saisir l'expérience, l'étude de la vie quotidienne est une approche des plus pertinentes. C'est au gré du quotidien que les individus expérimentent le monde et que se tisse l'expérience géographique. Par l'entremise des pratiques, l'étude de la vie quotidienne permet ainsi d'accéder à l'univers riche et dense du sens que les individus confèrent à leur milieu de vie. C'est dans ce milieu habité que s'articulent les pratiques communes et répétitives se produisant au fil des jours. En raison de leur caractère routinier, elles sont habituellement tenues pour acquises. L'observation des comportements (se déplacer, travailler, se divertir, socialiser...) et des lieux dans lesquels ils se produisent permet de s'attacher aux aspects visibles et concrets des rapports hommesmilieux. Mais ces actions sont révélatrices des motivations qui les guident, dans un espace traversé de nombreuses inégalités tant spatiales que sociales. L'analyse de la vie quotidienne met de l'avant les stratégies individuelles qui alimentent les pratiques (De Certeau, 1980; Goffman, 1973). Cela permet de dépasser leur matérialité pour accéder aux modalités par lesquelles les uns et les autres font leur place dans un environnement qui ne leur est pas toujours favorable (Harvey, 2003; Highmore, 2002, 2011 ; Lefebvre 1968, 2008).

Plusieurs géographes abordent l'expérience par l'entremise des espaces et des lieux que les individus 
pratiquent au quotidien, habitent, fréquentent et investissent, parce que "notre existence, à chaque instant et de bout en bout, est entièrement spatiale » (Lussault, 2007, 7). Les significations données aux espaces du quotidien se tissent au gré des pratiques produites dans des lieux tangibles, ce qui justifie leur observation fouillée, comme l'ont fait Gilbert et ses collègues dans l'espace transfrontalier d'Ottawa et de Gatineau (2014). Cette analyse révèle que les groupes minoritaires développent des stratégies selon le côté de la frontière duquel ils se situent. Les pratiques matérialisent leur résistance individuelle et collective devant la majorité et ouvrent la porte à la face cachée du quotidien.

Si le quotidien s'expérimente au moment présent vécu dans un lieu donné, la temporalité toujours en filigrane le structure. Au-delà des pratiques journalières, les représentations diachroniques et prospectives des lieux sont essentielles pour saisir l'expérience géographique. Le quotidien n'est pas uniquement journalier, il peut aussi bien être hebdomadaire, mensuel, annuel, voire se produire sporadiquement et ce, parce qu'il s'inscrit éminemment dans un cycle de vie particulier s'organisant autour des itinéraires de la vie quotidienne (Di Méo, 1999). Haicault insiste sur l'importance des événements imprévus ou spontanés venant déstabiliser le caractère « ordinaire » du quotidien, comme la venue d'un enfant ou un déménagement $(2000,22)$. À cela s'ajoutent les festivals, les fêtes ou les rites funéraires qui font aussi partie du quotidien. Ces passages empruntés à Tuan révèlent toute la richesse de la multitemporalité pour une meilleure compréhension de l'expérience : «The past really existed. All that we are we owe to the past. The present also has its merit; it is our experiential reality [...]. The future, in contrast, is a vision » $(2005,197)$.

En somme, les enseignements tirés de ces diverses perspectives pour la compréhension des relations homme-milieu sont à la fois riches et foncièrement complémentaires. Ainsi, il apparait que c'est sur l'élaboration d'un cadre conceptuel permettant l'articulation et la mise en relation entre les dimensions objectives et subjectives puis les dimensions individuelles et structurelles de l'expérience géographique que les efforts théoriques doivent porter.

\section{Grille D'ANALYSE DE L'EXPÉRIENCE GÉOGRAPHIQUE DE LA CAMPAGNE}

Une approche expérientielle de la campagne constitue le socle de cette grille d'analyse. Les dimensions retenues rassemblent sens des lieux, pratiques quotidiennes et habitus (figure 1). Notre approche s'appuie donc sur une micro-analyse sans toutefois négliger les milieux et les facteurs structuraux qui figurent à l'arrière-scène du quotidien. Elle s'inscrit dans une perspective qui prête à la vie quotidienne un rôle déterminant dans l'expérience que les individus entretiennent avec leur milieu de vie.

La grille d'analyse insiste sur la nécessité d'établir la relation étroite et réciproque d'une part, entre les dimensions individuelles et contextuelles, et d'autre part, entre celles-ci et l'expérience géographique. Le pointillé représente la possibilité d'inclure d'autres éléments, ce qui rappelle le caractère dynamique de l'expérience géographique. Les dimensions que sont les milieux et les facteurs structuraux sont localisées au haut et au bas du schéma pour illustrer combien elles encadrent, voire structurent l'expérience, sans exclure l'ajout éventuel d'autres éléments contextuels.

\section{Dimensions individuelles de l'expérience géographique}

\section{Sens des lieux}

Le sens des lieux représente les rapports subjectifs et affectifs que les ruraux entretiennent avec la campagne et les populations qui l'habite. Nous l'appréhendons par leurs représentations qui renvoient aux formes symboliques entourant leurs schèmes de pensée, leur sentiment d'appartenance à leur milieu de vie, ainsi que leurs identités territoriales révélées par leur autodéfinition. Le sens des lieux dérive de différentes modalités d'appartenance qui impliquent également des conditions sociales déterminantes pouvant créer des clivages ainsi que des intérêts et des valeurs partagés par un groupe. Ainsi, cette grille d'analyse s'inscrit au sein des travaux en sciences régionales et en développement territorial qui accordent une importance centrale à la subjectivité humaine, à l'instar de plusieurs contributions scientifiques (Bassand, 2002; Dionne et Thivierge, 2000 ; Faure, 1995; Mathieu, 1989; Moquay, 1997 ; Paasi, 2002; Raagmaa, 2002; Sencébé, 2011). C'est 


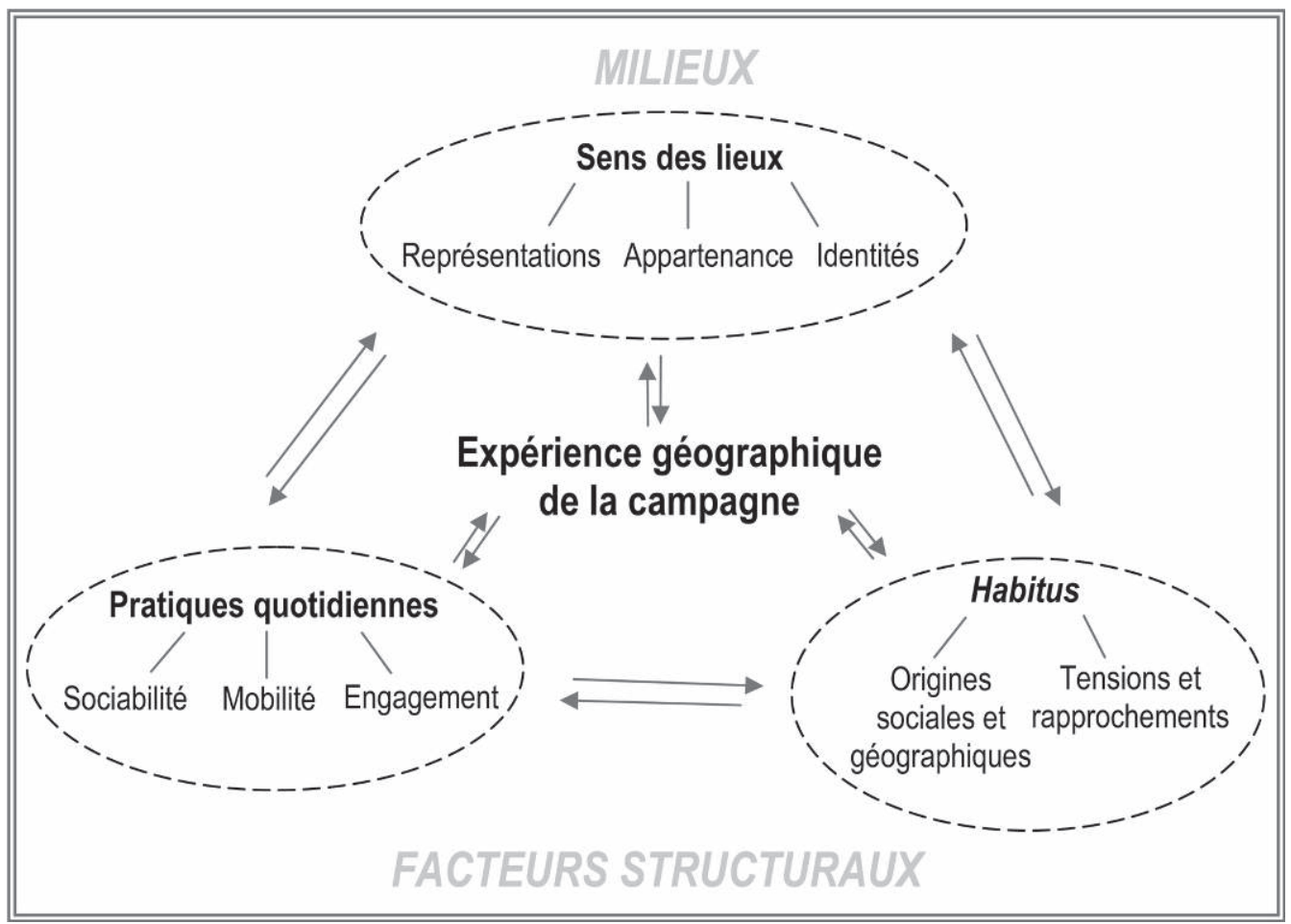

Figure 1 : Grille d'analyse de l'expérience géographique de la campagne Analytical framework of the geographical experience of the countryside

par l'entremise du sens des lieux qu'il est possible d'explorer la campagne vécue.

Les rapports aux lieux sont intimement liés aux représentations idéelles et symboliques entretenant une relation directe et réciproque avec les comportements. Elles sont fondamentales pour mettre en lumière l'expérience géographique. Pour Tuan, l'expérience "is compounded of feeling and thought » $(2005,10)$. La campagne n'est pas uniquement un milieu physique et social avec des attributs spécifiques, elle est chargée de significations et de connaissances acquises par l'expérience.

\section{Pratiques quotidiennes et habitus}

L'expérience se produit dans des lieux concrets dans lesquels se matérialisent différentes pratiques quotidiennes loin d'être anodines. En effet, « pratiquer les lieux, c'est en faire l'expérience, c'est déployer, en actes, un faire qui a une certaine signification » (Stock, 2004). Les pratiques se déroulent dans l'espace local, régional, extrarégional et le cyberespace. Dans les campagnes québécoises, elles sont particulièrement façonnées par la dispersion et l'éloignement. Les pratiques de mobilité vers les villes modulent spécialement l'expérience de la ruralité. Cela parce que la mobilité comporte des facettes objective (fréquence, durée) et subjective (valeurs, aspirations, émotions) (Cresswell, 2010).

Non seulement les pratiques quotidiennes sontelles localisées dans des lieux concrets à diverses échelles, mais elles cristallisent des liens et des interactions se construisant au sein du voisinage, de la famille, du réseau d'amis, des espaces professionnel, associatif ou politique. Par l'entremise des pratiques d'engagement, il est possible d'entrevoir jusqu'à quel point les ruraux interagissent avec l'ensemble de la population locale et régionale et s'investissent dans leur milieu de vie.

Les pratiques quotidiennes permettent d'étudier les liens de sociabilité entre les populations rurales qu'elles soient nouvelles ou anciennes puisque l'expérience géographique est fondamentalement sociale. L'individu est non seulement en interaction avec les lieux qu'il fréquente, mais aussi en relation avec les autres qu'il côtoie dans ces mêmes lieux. Indubitablement spatialisées, ces interactions quotidiennes sont modulées par les trajectoires de vie, les origines géographique et sociale, les pratiques, les représentations, les personnalités de chacun (Di Méo et Buléon, 2005). De ces différences naissent des tensions dans lesquelles peuvent se dessiner des 
rapports de pouvoir. Les formes de sociabilité les plus banales, comme saluer son voisin, "sont porteuses de fortes charges symboliques qui structurent des jeux de position, si bien que leur étude permet de dresser la carte des différenciations sociales » (Lévy et Lussault, 2003, 849). L'expérience se matérialise dans un espace social où se rencontrent, se confrontent, se mobilisent et peuvent se rallier les individus, un espace «médiateur des relations sociales »(Chivallon, 2000). De l'espace de rencontre où se côtoient les différentes populations rurales, nouvelles comme anciennes, émanent tensions, conflits, compromis et nouvelles alliances.

Les pratiques sont façonnées par les groupes d'appartenance des ruraux qui découlent, entre autres, de leur classe sociale, leur trajectoire géographique (lieu de jeunesse, lieu de vie adulte, migration...), leur statut socioprofessionnel, leur âge... En outre, les microhistoires individuelles agissent sur les rapports à l'Autre, dont les types de personnes fréquentées, les affinités et la nature des liens de sociabilité entretenus avec eux. Dans le contexte des interactions quotidiennes, l'habitus est un facteur explicatif de l'expérience géographique permettant de contextualiser l'individu en tant qu'être social. Il dévoile l'effet de l'origine urbaine ou rurale ou de classe sociale quant aux tensions liées aux représentations et usages de la campagne. Sans quili en soit forcément conscient, ses façons d'agir, de penser et de concevoir le monde sont conditionnées par les structures objectives et subjectives qui lui ont été transmises ou qu'il a acquises dans le passé, que ce soit par les parents ou par la scolarisation (Bourdieu, 1979) ou encore au cours de la trajectoire géographique.

L'habitus occupe un rôle déterminant dans l'expérience des lieux et occasionne des luttes matérielles et symboliques que nous observons à la campagne. À titre d'exemple, les liens sociaux parfois élitistes des nouveaux ruraux appartenant à la bourgeoisie, leurs goûts et leurs pratiques de consommation d'arts et de culture ou de produits raffinés configurent leur expérience rurale ${ }^{2}$. L'ensemble des dispositions propres aux néo-ruraux a été bien documenté dans les travaux sur l'embourgeoisement rural qui décrivent souvent ces individus en faisant réfé-

2. Les populations néo-rurales sont toutefois très hétéroclites et plusieurs nouveaux ruraux ne font pas partie d'une bourgeoisie ex-urbaine. On n'a qu'à penser aux migrants de classes plus modestes qui s'établissent à la campagne pour accéder à un logement moins coûteux ou pour des raisons professionnelles ou familiales (Simard et Guimond, 2009). rence à leur classe sociale plus aisée que celle des ruraux de longue date (Cloke et al., 1998; Guimond et Simard, 2010; Stockdale, 2010). Peuvent alors surgir des clivages liés à des conditions sociales divergentes (réseaux, niveau de scolarité, catégorie socioprofessionnelle, prise de parole en public...). L'habitus permet, en se basant sur les schèmes référentiels et les pratiques, de rendre compte des différences dans les modes de vie, valeurs, opinions, représentations et identités propres à chacun. Ces repères découlent d'une origine sociale alimentée par les milieux géographiques où ils furent acquis ou transmis antérieurement (Marshall et Foster, 2002). L'expérience géographique ne peut être détachée des «milieux où on a vécu, leurs caractéristiques sociales et spatiales, ainsi que [...] les routines spatiotemporelles et les significations qui y sont attachées » (Fortin et Després, 2008, 155). Ainsi, elle est façonnée tant par les conditions sociales que les expériences géographiques du passé.

\section{Dimensions contextuelles de l'expérience géographique}

\section{Milieux}

L'expérience géographique s'opère dans un milieu avec ses propres conditions d'existence liées à un contexte géographique particulier. La campagne s'organise en fonction des possibilités d'emplois et du potentiel de projets entrepreneurial, de la localisation par rapport aux centres urbains, des infrastructures et des services locaux, des modalités de la planification et de l'aménagement du territoire, de la réglementation de zonage... Kayser soulève, dans ses recherches sur la renaissance rurale en France, que «l'évolution des territoires se différenciera en fonction de leur position dans le contexte du peuplement, des moyens de communication, des activités économiques ou du semis urbain » (1993). Chaque milieu a sa propre dynamique d'ouverture et de fermeture, de tradition d'accueil ou de guerre de clocher...

Dans le contexte de la migration de la ville vers les campagnes, notamment au sein des migrations d'agrément (Moss, 2006), les caractéristiques paysagères (Ruiz et Domon, 2013) sont un important facteur attractif du milieu. Au Québec comme ailleurs, les municipalités les plus convoitées sont celles dont 
les paysages sont les plus attrayants. Mais ne tombons pas dans les clichés faciles. Les municipalités moins attrayantes aux yeux d'un certain type d'individu peuvent l'être pour d'autres, comme celles où le prix des terrains et des maisons est abordable et qui accueillent des migrants en situation précaire (Cognard, 2010).

Dans notre grille d'analyse, la notion de milieux est écrite au pluriel, car l'expérience de la campagne n'est pas restreinte à un seul milieu. On y compte les villages environnants, les villes régionales ou les métropoles localisées tant à proximité du domicile qu'ailleurs dans la province, le pays, le monde. L'espace virtuel fait partie du quotidien des ruraux alors que les technologies de l'information et des communications (TIC) sont fréquemment utilisées pour l'emploi, les réseaux sociaux, les actualités, la consommation, les loisirs... Multiscalaire et modulée par la mobilité, la vie quotidienne à la campagne ne peut se fermer à l'expérience qui se produit audelà de ses frontières, dans l'espace " extrarural » (Halfacree, 2007).

\section{Facteurs structuraux}

L'expérience géographique évolue par ailleurs dans un contexte sociétal plus large que nous nommons facteurs structuraux. Dans les campagnes, on n'a qu'à penser au désengagement de l'État-providence, à la crise financière qui se reflète sur le budget des municipalités, à la décentralisation des lieux de travail, à la migration de la ville vers la campagne, à la difficulté accrue de l'accès à la propriété dans certaines campagnes convoitées, à la dévitalisation du milieu rural et au vieillissement de sa population, au rejet de la société de surconsommation ou à la montée des valeurs environnementalistes. Ajoutons que l'amélioration des réseaux de transport et des TIC, rendant les lieux constitutifs de la vie quotidienne plus accessibles physiquement ou virtuellement, agissent directement sur le rapport au temps et à l'espace des ruraux. Ceci est crucial dans un milieu où les distances sont, de tout temps, un enjeu. Bien que les manifestations structurelles ne soient pas toujours immédiates et visibles, il importe de rester sensible à leur entrecroisement pour une meilleure compréhension de l'expérience de la campagne.

\section{Cas-type de Léo}

Afin de tester cette grille d'analyse, nous présentons le cas-type de Léo ${ }^{3}$. Celui-ci a été sélectionné à partir d'un échantillon de 71 entrevues qualitatives approfondies menées en 2006 et 2007 dans les municipalités régionales de comtés (MRC) de Brome-Missisquoi et d'Arthabaska auprès de nouveaux résidents et de ruraux de longue date ${ }^{4}$. Ce cadre géographique a déjà été décrit ailleurs (Simard et Guimond, 2010)5 . Bien que Léo ait été retenu du fait que son expérience recoupait celle d'autres nouveaux résidents, les expériences de chacun de ces participants sont manifestement singulières et distinctes, les ruraux, nouveaux comme anciens, formant une population hétérogène, à l'instar des milieux différenciés qu'ils habitent. Le but de cette partie n'est pas de brosser un portrait général de ces populations, ni de l'expérience géographique des campagnes québécoises, mais plutôt d'illustrer la capacité opératoire et le potentiel de la grille d'analyse proposée.

Léo se situe dans la trentaine et il est père de trois enfants. Les professions de ses parents, tous deux travailleurs sociaux dans le secteur public, ainsi que son profil (éducation, revenu, goûts, valeurs, intérêts, loisirs, etc.), révèlent qu'il provient d'une classe sociale moyenne se situant dans une position intermédiaire entre les classes populaires et supérieures. Il a grandi et a fait ses études dans un quartier ouvrier de Montréal que ses parents habitent toujours. Léo détient deux diplômes d'études collégiales, l'un dans un métier, l'autre dans les arts. N'étant pas rectiligne, sa trajectoire professionnelle correspond à celle des jeunes adultes d'aujourd'hui puisque, depuis son entrée sur le marché du travail, il a occupé plusieurs emplois, souvent de « survie »,

3. Pour conserver l'anonymat du participant, un nom fictif lui a été désigné. Toute information permettant de le reconnaître n'est pas dévoilée, dont le nom de la municipalité qu'il habite. Précisons qu'il réside dans une municipalité de moins de 1000 habitants faisant partie de la MRC d'Arthabaska dont l'économie repose surtout sur les secteurs agricole, industriel et manufacturier.

4. Dans le cadre de cette recherche, les néo-ruraux sont des ex-urbains qui se sont installés en permanence à la campagne depuis un minimum d'un an et un maximum de 20 ans. Les ruraux de longue date sont des individus nés à la campagne et qui y vivent depuis toujours malgré des migrations temporaires ou des ruraux qui y résident en permanence depuis plus de vingt ans sans y être nés. Un nouveau résident est sélectionné ici plutôt qu'un rural de longue date car la recherche vise à cerner le phénomène de néo-ruralité au Québec.

5. Se référer également aux travaux du Groupe de recherche sur la migration ville/campagne et les néo-ruraux, dirigé par Myriam Simard de l'INRS [http://www.neoruraux.ucs.inrs.ca/]. 
dans des secteurs n'étant pas liés à sa formation académique. Léo se dit polyvalent, débrouillard, flexible et curieux, ce qui est conforté par son parcours scolaire et ses expertises professionnelles et personnelles diversifiées. Le revenu familial annuel brut du ménage de Léo, en 2005, se situait entre 30000 et 45000 \$. C'est bien en deçà du revenu moyen des ménages québécois en couple avec enfants qui s'élevait, pour la même année, à 70106 \$ (Institut de la statistique du Québec, 2014). Cette différence notable s'explique entre autres par le faible revenu de la femme de Léo, qui s'occupe des enfants en bas âge et de la maison.

Avant de s'installer en permanence à la campagne il y a quatre ans, au moment de l'entrevue, Léo a vécu la majeure partie de sa vie à Montréal, bien que celle-ci fût entrecoupée de voyages en Europe et en Amérique du Sud, ainsi que dans plusieurs états américains ${ }^{6}$. Voyageur dans l'âme, il est toujours en quête de nouveaux défis et d'aventures. C'est d'ailleurs une des principales raisons pour laquelle lui et sa conjointe ont fait le choix de migrer à la campagne, un milieu de vie qu'ils n'avaient guère connu avant leur migration. Voulant " essayer quelque chose de nouveau », ils étaient à la recherche de grands espaces, de tranquillité et de nature pour y élever leurs enfants. On voit ici à l'œuvre l'effet structurel du contexte sociétal plus large où la recherche d'une qualité de vie domine. D'ailleurs, les représentations de la campagne de Léo correspondent à celles souvent projetées dans les médias et l'opinion publique qui présentent la campagne comme un "refuge » permettant de fuir les inconvénients urbains. Ces représentations véhiculent l'idée que la campagne offre une qualité de vie d'une valeur telle qu'on ne saurait l'estimer. Avant de s'y installer, Léo considérait que campagne rimait avec «tranquillité », " sécurité » et « espace». La plupart de ses représentations antérieures concordent toujours avec celles qu'il a aujourd'hui. Toutefois, cherchant un équilibre « famille-boulotloisirs-amis » lui permettant de réaliser ses rêves, Léo est « désillusionné » du marché de l'emploi en région.

L'expérience quotidienne de Léo est profondément marquée par l'absence d'un emploi convenable dans son nouveau milieu de vie. Lors de ses

6. La durée totale de ces séjours à l'étranger dépasse quatre ans. deux premières années d'établissement, Léo dénicha quelques emplois localement, mais ceux-ci étaient précaires et n'offraient pas de bonnes conditions (faible rémunération, irrégularité du nombre d'heures de travail, incertitude face à l'avenir, etc.). Le travail qu'il occupe actuellement lui procure un meilleur salaire, mais sa qualité de vie en est grandement affectée :

"On n'a pas eu de difficultés depuis notre arrivée en permanence à la campagne, à part pour trouver des emplois. Je dois travailler à Montréal, Québec, Trois-Rivières..., où se trouvent les contrats de l'entreprise qui m'embauche. Normalement je pars le lundi matin et je reviens le vendredi soir et je suis en vacances ou au chômage aléatoirement, entre les contrats. [...] Il faut que je change encore d'emploi parce que j'ai trois enfants : partir le lundi matin et revenir le vendredi soir, ce n'est pas l'idéal, je ne suis pas là finalement. »

Afin de subvenir aux besoins de sa famille, il doit, depuis deux ans, s'exiler hebdomadairement dans diverses localités québécoises pour réaliser des contrats temporaires et irréguliers pour l'entreprise qui l'embauche. Tant avec cet emploi qu'avec ceux qu'il a occupés localement, Léo fut dans l'obligation d'avoir recours à l'assurance-emploi :

"Je n'ai jamais eu de chômage avant de partir de Montréal, mais depuis que je suis ici, les trois emplois que j'ai occupés m'ont mis sur le chômage éventuellement, c'est soit des emplois saisonniers ou contractuels. [...] Chaque année, j'ai une période de chômage qui est tout à fait contre mon gré. "

Ceci dénote le problème structurel du manque d'emplois qualifiés dans sa localité et en région et potentiellement de la reconnaissance des diplômes et des expertises. Léo explique que les employeurs de son milieu privilégient l'embauche de résidents de longue date nés dans Arthabaska. Son statut de nouveau résident est donc un obstacle à son insertion professionnelle puisque son réseau et ses repères sociaux comme spatiaux découlant de son habitus ne se trouvent pas dans son nouveau milieu de vie.

Déplorant son manque de temps avec sa famille, il envisage de changer d'occupation à nouveau. Pour remédier à cette situation, Léo et sa conjointe affectionnent l'idée de créer leurs propres emplois 
car le travail autonome leur permettrait d'avoir une meilleure qualité de vie conciliant famille, travail et intérêts personnels. Dans leur projet, on décèle les valeurs dominantes des jeunes adultes d'aujourd'hui, où la volonté d'accéder à une meilleure qualité de vie passe par la réalisation de soi et par un équilibre travail-famille. L'absence d'un réseau Internet à large bande dans sa localité est un sérieux obstacle à leur démarche. Ainsi, les facteurs structuraux comme les valeurs sociétales contemporaines, les contraintes du marché de l'emploi en régions et l'état lacunaire des infrastructures de TIC agissent directement sur le quotidien de Léo.

La situation professionnelle de Léo teinte éminemment son rapport subjectif avec la campagne. Il en fait mention tout au long de son récit, même lorsqu'il décrit son identité territoriale et le sens qu'il confère à son milieu de vie :

«Notre obstacle ici, c'est vraiment l'accessibilité à l'emploi. [...] On est ici, on aime ça, mais je ne travaille pas ici, mon job est ailleurs. Est-ce que je suis vraiment un gars de la campagne? Oui, je vis ici, mais ce n'est pas la campagne qui me fait vivre. »

Léo est moyennement attaché à son milieu, sentiment qu'il explique par son tempérament. Il dit ne s'être jamais attaché à un milieu, ne voulant pas « être triste de s'en séparer ». D’ailleurs, Léo et sa conjointe n'excluent pas l'idée de partir éventuellement. Ils ne savent pas où ils iraient, ni à quel moment s'effectuerait leur départ qui semble être motivé par leur souci d'offrir une meilleure éducation à leurs enfants, tout en leur donnant accès à la diversité urbaine. Leur profond désir de vivre de nouveaux défis anime leurs projets d'avenir :

«La campagne nous satisfait pour l'instant et [correspond à] notre dose d'aventure qu'on est capable d'avoir avec les enfants. Mais un moment donné, ça ne sera plus assez, nous allons vouloir une plus forte dose d'aventure, de dépaysement. Nous, on ne cherche pas à s'entourer de ce qu'on connaît, on cherche à modifier et élargir un peu notre horizon puis maintenant celle des petits. "

En dépit de sa situation professionnelle difficile, Léo souligne s'être bien intégré socialement. Ses premiers emplois locaux lui ont permis de nouer des liens rapidement puisqu'il devait côtoyer quotidiennement les résidents de sa municipalité. Son ouverture aux autres a facilité son insertion sociale. Il se sent bien dans sa localité, même s'il sait qu'aux yeux des résidents de longue date, il restera toujours « le gars qui ne vient pas d'ici »:

"Pour nous, ça n'a pas été trop difficile de se faire un nowveau réseau social, mais je pense qu'on est de nature ouverte à tout, puis à tout essayer. Par contre, on est étranger pour encore 10 ans, 20 ans : Ah oui! Le gars qui ne vient pas d'ici. Même dans 20 ans, je suis sûr que si je suis encore là, ça va être ça. Puis même mes enfants, [les gens vont savoir] qu'ils se sont installés ici : Oui, oui, ton père venait de Montréal. Mais sinon, on s'est bien intégré quand même malgré ça.»

Léo a développé des liens d'amitié à la campagne, surtout avec des personnes se situant à la même étape de vie que lui et sa famille. Les activités sociales qu'il réalise avec eux s'expriment sous forme de sorties avec les enfants, repas, participation à des événements locaux, etc. Il entretient également des relations cordiales et d'entraide avec ses voisins qui sont des résidents de longue date. Léo fréquente surtout des ruraux de longue date nés dans sa municipalité. Ses amis intimes sont d'ailleurs un jeune couple de ruraux de longue date qu'il connaissait avant même de migrer, ainsi que des amis plus anciens résidant toujours à Montréal. Ses pratiques de sociabilité montrent la juxtaposition de deux univers à la fois rural et urbain faisant tous deux partie de son expérience géographique. En expliquant les modalités de son insertion sociale, Léo déplore le manque de lieux de sociabilité à l'échelle locale, ce qui contribue à l'isolement de certaines personnes, dont les « jeunes mamans à la maison » comme sa femme. Il souhaite que son milieu puisse lui offrir des lieux de socialisation (restaurant, café, petit bistro). Son besoin d'accéder à un univers social plus diversifié, tout en bénéficiant d'une variété de produits et de services, est assurément influencé par ses expériences urbaines antérieures et actuelles.

Effectivement, Léo entretient un rapport étroit avec son lieu d'origine, Montréal, parce qu'il doit s'y rendre à l'occasion pour travailler et pour visiter sur une base régulière famille et amis. Il en profite pour «faire le plein de ce qu'il a besoin et qu'il ne peut pas trouver à la campagne ». Cela n'empêche pas qu'il fréquente hebdomadairement la principale ville régionale desservant sa MRC pour faire 
les courses. Son expérience de la campagne n'est donc pas confinée à son milieu de vie immédiat, mais elle est grandement marquée par les pratiques de mobilité intra et extrarégionales, parfois choisies, d'autres fois contre son gré.

Léo prend part régulièrement à des festivals, foires ou autres événements dans sa municipalité et dans sa MRC. Il utilise les infrastructures locales, dont la patinoire avec les enfants. Il n'a pas le temps de s'impliquer bénévolement en raison de ses charges professionnelles et familiales, puis ses nombreux déplacements à l'extérieur. Léo s’intéresse néanmoins aux enjeux touchant son milieu de vie. Il précise que la qualité et l'approvisionnement en eau sont actuellement des enjeux locaux importants. Il s'exprime aussi sur la question des productions porcines qui pose de nombreux défis aux décideurs et aux citoyens de sa municipalité. Selon lui, les décideurs se trouvent devant un dilemme et ils devront éventuellement faire un choix qui déterminera l'avenir de sa municipalité : développer l'agriculture intensive ou miser plutôt sur le secteur touristique. En partageant sa vision d'avenir, Léo insiste sur le repeuplement de son milieu qui pourrait être assuré par la migration d'urbains vers la campagne :

"Moi, la tendance que je vois est qu'il va y avoir de plus en plus de monde, comme nous, qui vont partir des gros centres et qui vont chercher à être tranquilles. [...]. C'est une qualité de vie, juste l'air qu'on a, la paix. C'est un beau coin ici. Puis tout ça va amener d'autres choses qui manquent dans la région: plus il va y avoir de monde, plus il va y avoir de besoins, puis des gens qui vont offrir des services pour combler ces besoins-là. [...] Ces gens qui arrivent de l'extérieur vont créer leur emploi et ils vont peut-être engager des gens. Je pense que c'est ça l'avenir.»

Les représentations prospectives de Léo laissent présager la possibilité d'une amélioration sur le plan des emplois en région, ce qui pourrait avoir un réel impact sur sa situation professionnelle actuelle ainsi que celle de sa conjointe. Les représentations diachroniques des lieux alimentent le quotidien de Léo.

\section{EN GUISE DE CONCLUSION : LA PORTÉE DE LA GRILLE D'ANALYSE}

Le cas-type de Léo illustre à quel point l'articulation des pratiques, du sens des lieux et de l'habitus est au cœur de l'expérience géographique de la campagne. Or, cette dernière est structurée par les contraintes du marché de l'emploi local engendrant une mobilité non désirée ainsi que par les valeurs actuelles des jeunes adultes (quête de nouveaux défis, d'une meilleure qualité de vie, d'un équilibre travail-famille...). Chacune des dimensions observées peut avoir un poids différent qui varie selon l'individu et plus largement selon son milieu, comme le démontrent nos analyses antérieures sur l'habitus (Guimond et al., 2014) ainsi que sur le sens des lieux et les pratiques d'engagement local (Guimond et Simard, 2011). L'expérience géographique est unique à chacun et nécessairement multiforme. Nous reconnaissons les limites de notre démarche méthodologique s'appuyant sur la présentation d'un seul cas d'étude. Cependant, cette grille d'analyse peut éventuellement contribuer à de nouvelles typologies basées sur l'expérience de la campagne. C'est à partir de ces expériences individuelles que se dessine la ruralité contemporaine.

Cette grille d'analyse permet de mettre en relation plusieurs facettes, tant individuelles que contextuelles, de l'expérience géographique. Dans la foulée des travaux géographiques sur la déconstruction de la lecture binaire des espaces et des groupes sociaux, elle suggère de nouvelles façons d'habiter et de cohabiter dans ce milieu qu'est la campagne. En s'appuyant sur les mécanismes à l'œuvre dans les lieux quotidiens de sociabilité, ce cadre peut apporter de nouvelles connaissances sur les modalités de la rencontre des diverses populations rurales.

Plus concrètement pour les communautés rurales, cette lecture de l'expérience des campagnes permet aux acteurs locaux de mieux cerner comment les ruraux expérimentent, pratiquent et se représentent la campagne, à savoir celle d'hier, d'aujourd'hui et de demain. Dans une perspective de développement territorial et de volonté de renouvellement démographique, cela fait ressortir tant les faiblesses que les forces des milieux ruraux ainsi que leurs facteurs d'attractivité et de rétention. Ceci est utile pour élaborer des stratégies et des politiques dans le but d'attirer de nouvelles populations potentielles et/ou 
d'accueillir, d'intégrer et de retenir celles qui y sont déjà. Notre approche incite à reconnaitre la complexité des pratiques quotidiennes de mobilité et de sociabilité, des représentations et des identités qui se dessinent désormais à la campagne.

De façon plus générale, cette démarche offre la possibilité d'identifier les impacts et défis liés à la diversification des populations rurales, dont les nouveaux liens de sociabilité qui en émergent au quotidien. En faisant valoir les expériences polymorphes de la campagne, elle renforce l'idée qu'il faut dépasser la catégorisation dichotomique traditionnelle des populations selon leurs origines rurale ou urbaine et entrevoir plutôt la construction d'une nouvelle ruralité marquée par leur rencontre. Ces catégories sont trop restrictives, puisque chacun a ses propres visées pour ce milieu de vie qu'est la campagne, selon qu'il soit jeune ou plus âgé, travailleur ou retraité, agriculteur ou artiste, décideur ou simple citoyen, bien nanti ou défavorisé... De par la diversité des populations rurales, établir des références communes à la campagne est de plus en plus difficile. Les visées et les tensions qui peuvent en émerger s'inscrivent dans des processus complexes que l'expérience géographique permet d'élucider.

Ainsi, cette démarche suscite des réflexions critiques et prospectives sur l'évolution et le sens des transformations que connaissent les campagnes contemporaines. Cette grille d'analyse peut s'appliquer à divers milieux, d'autres espaces, d'autres populations que ceux illustrés ici, tant et aussi longtemps que leurs propres spécificités soient prises en compte. L'expérience géographique, telle qu'elle se décline au quotidien, offre des repères cruciaux pour conceptualiser et repenser de façon plus interactive les relations des hommes aux milieux qu'ils habitent.

\section{Remerciements}

Je remercie vivement Anne Gilbert et Myriam Simard pour leurs commentaires constructifs et leurs relectures critiques. Merci également à Nicole Mathieu, Yves Jean Mario Bédard ainsi qu'aux évaluateurs anonymes pour leurs judicieux commentaires. La grille d'analyse qui y est présentée a fait l'objet de communications auprès des pairs lors d'évènements scientifiques en France et au Canada, et les échanges tenus lors de ces événements ont permis son affinement. Cette recherche a bénéficié du soutien financier $d u$ Conseil de recherches en sciences humaines $d u$ Canada (CRSH).

\section{Bibliographie}

Bassand M., 2002. L'identité et le sens du développement territorial, in GRIDEQ, Le développement des territoires : nouveaux enjeux, Rimouski, UQAR et GRIDEQ, p. 15-26.

BÉdARD M., 2011. Résonances à L'homme et la Terre d'Éric Dardel dans la géographie culturelle québécoise, Cahiers de géographie du Québec, vol. 55, n 155, p. 279-291.

Bourdieu P., 1979. La distinction. Critique sociale du jugement, Paris, Les Éditions de Minuit, 672 p.

Buttimer A., 1969. Social space in interdisciplinary perspective, The Geographical Review, vol. 59, p. 417-426.

Chivallon C., 2000. D'un espace appelant forcément les sciences sociales pour le comprendre, in LÉvy J. et LusSault M. (dir.), Logiques de l'espace, esprit des lieux. Géographies à Cerisy, Paris, Belin, p. 299-317.

Cloke P., Phillips M., Thrift N., 1998. Class colonisation and lifestyle strategies in Gower, in Boyle P.J. et HalfaCREe K. (dir.), Migration into Rural Areas: Theories and Issues, Chichester, Wiley, p. 166-185.

Cognard F., 2010. "Migrations d'agrément » et nouveaux habitants dans les moyennes montagnes françaises : de la recomposition sociale au développement territorial. L'exemple $d u$ Diois, du Morvan et du Séronais, thèse de doctorat en géographie, Clermont université/université Blaise-Pascal, Clermont-Ferrand, $528 \mathrm{p}$.

Cresswell T., 2010. Towards a politic of mobility. Environment and Planning D: Society and Space, vol. 28, p. 17-31.

Dardel E., 1952. L'homme et la Terre, Paris, PUF, 133 p.

De Certeau M., 1980. L'invention du quotidien, 1. Ars de faire, France, Union Générale d'éditions (coll. 10-18), 349 p.

Dionne H., Thivierge N., 2000. Le développement humain et l'identité territoriale et communautaire, in CARRIER M., Côté S. (dir.), Gouvernance et territoires ruraux. Éléments d'un débat sur la responsabilité du développement, Sainte-Foy, Presses de l'université du Québec, p. 301-316.

Di Méo G., 1999. Géographies tranquilles du quotidien. Une analyse de la contribution des sciences sociales et de la géographie à l'étude des pratiques spatiales, Cahiers de géographie du Québec, vol. 43, n 118, p. 76-93.

Di Méo G., 2000. Que voulons-nous dire quand nous parlons d'espace? in LÉvy J., Lussault M. (dir.), Logiques de l'espace, esprit des lieux. Géographies à Cerisy, Paris, Belin, p. 37-48.

Di Méo G., Buléon P., 2005. L'espace social. Lecture géographique des sociétés, Paris, Armand Colin/VUEF, 304 p.

Domon G., Ruiz J., Paquette S., Roy L. 2011 . La recomposition sociodémographique des campagnes québécoises à la croisée des dynamiques agricoles et paysagères, Revue canadienne de développement régional, Vol. 34, n 4, p. 115-133. 
FAure A., 1995. Identité locale et développement rural. Le « pays » à l'épreuve du monde, in SAEZ J.-P. (dir.), Identités, cultures et territoires, Paris, Desclée de Brouwer, p. 145-154.

Fortin A. et Després C., 2008. Le juste milieu : représentations de l'espace des résidants du périurbain de l'agglomération de Québec, Cahiers de géographie du Québec, vol. 52, $n^{\circ} 146$, p. 153-174.

Frémont A., 1974. Recherches sur l'espace vécu, L'Espace géographique, vol. 3, p. 231-238.

Frémont A., 1976. La région, espace vécu, Paris, PUF, 223 p.

Gilbert A., Veronis L., Brosseau M. et Ray B., 2014. La frontière au quotidien. Expérience des minorités à Ottawa-Gatineau, Ottawa, Les Presses de l'Université d'Ottawa, 392 p.

Goffman E., 1973. La Mise en scène de la vie quotidienne 1: La présentation de soi, Paris, Les Éditions de Minuit, 256 p.

Guimond L., 2012. Lorsque les nouvelles populations rurales rencontrent les plus anciennes : l'expérience géographique au cœur de la nouvelle ruralité au Québec. Thèse de doctorat en géographie, université d'Ottawa, $210 \mathrm{p}$.

Guimond L., Gilbert A. et Simard M., 2014. Faire sa place et être de la place : la rencontre timide des nouveaux ruraux et des populations plus anciennes au Québec, Le Géographe canadien, vol. 58, $\mathrm{n}^{\circ} 1$, p. 123-139.

Guimond L., Simard M., 2010. Gentrification and neo-rural populations in the Québec countryside: representations of various actors, Journal of Rural Studies, vol. 26, p. 449-464.

Guimond L., Simard M., 2011. Les néo-ruraux et les ruraux de longue date sont-ils si différents au Québec? Analyse de leur mobilité, sens des lieux et engagement, Revue canadienne des sciences régionales, vol. 34, n 4, p. 151-163.

Haicault M., 2000. L'expérience sociale du quotidien : corps, espace, temps, Ottawa, Les Presses de l'Université d'Ottawa, $224 \mathrm{p}$.

Halfacree K., 2007. Trial by space for a "radical rural": Introducing alternative localities, representations and lives, Journal of Rural Studies, vol. 23, p. 125-141.

Harvey D., 2003. Paris, Capital of Modernity, New York/London, Routledge, $372 \mathrm{p}$.

Highmore B., 2002. Introduction. Questioning everyday life, in B. Highmore (dir.), The everyday life reader, London, Routledge, p. 1-33.

Highmore B., 2011. Ordinary lives: Studies in the everyday, New York, Routledge, 195 p.

Institut de la statistique du Québec, 2014. Le Québec chiffres en main, Québec, Institut de la statistique du Québec. $72 \mathrm{p}$.

JEAN B., 2006. Les études rurales québécoises entre les approches monographiques et typologiques, Recherches sociographiques, vol. XLVII, p. 503-529.

JeAn B., Lafontaine D. (dir.), 2010. La multifonctionnalité de l'agriculture et des territoires ruraux. Enjeux théoriques et d'action publique, Rimouski, CRDT et GRIDEQ, 228 p.

Jean B. en collaboration avec Desrosiers L., Dionne S., 2014. Comprendre le Québec rural, 2e éd., Université du Québec à Rimouski - Chaire de recherche du Canada en développement rural - GRIDEQ - CRDT, 166 p..
KaYser B. (dir.), 1993. Naissances de nouvelles campagnes, Marseille, Éditions de l'Aube/DATAR, 174 p.

Lefebvre H., 1968. La vie quotidienne dans le monde moderne, Paris, Gallimard, 383 p.

Lefebvre H. 2008. [1947]. Critique of everyday life, vol. 1, London and New York, Verso, 283 p.

LÉvy J., Lussault M., 2003. Dictionnaire de la géographie et de l'espace des sociétés, Paris, Belin, 1034 p.

Lussault M., 2007. L'homme spatial. La construction sociale de l'espace humain, Paris, Éditions du Seuil, 366 p.

Marshall J., Foster N. 2002., "Between Belonging": Habitus and the migration experience, The Canadian Geographer, vol. 46, $\mathrm{n}^{\circ} 1$, p. 63-83.

Mathieu N., 1989. Solidarité, identité, innovation, les tensions fondatrices de la société méjanaise, Annales du Parc national des Cévennes, tome 4, p. 229-261.

Mathieu N., 2007. L'évolution des modes d'habiter : un révélateur des mutations des sociétés urbaines et rurales. Introduction, in LuginbüHL Y. (dir.), Nouvelles ruralités, nowvelles ruralités en Europe, Bruxelles/Bern/Berlin/Frankfurt am Main/New York/Oxford/Wien, Peter Lang, p. 25-45.

Mathieu N., 2014. Mode d'habiter : un concept à l'essai pour penser les interactions hommes-milieux, in Chenorkian R., Robert S. (dir.), Les interactions hommes-milieux. Questions et pratiques de la recherche en environnement, Versailles, Quæ, p. 97-130.

Moquay P., 1997. Le sentiment d'appartenance territoriale, in Gauthier M. (dir.), Pourquoi partir? La migration des jeunes d'hier et d'aujourd'hui, Sainte-Foy, Les éditions de l'IQRC, p. 243-256.

Moss L. A. G., (dir.), 2006. The amenity migrants: Seeking and sustaining mountains and their cultures, Oxfordshire and Cambridge, CAB International, 329 p.

PAAsi A., 2002. Bounded spaces in the mobile world: deconstructing "regional identity", Tijdschrift voor Economische en Sociale Geografie, vol. 93, n² 2, p. 137-148.

Raagmaa G., 2002. Regional Identity in Regional Development and Planning, European Planning Studies, vol. 10, $\mathrm{n}^{\circ} 1$, p. $55-76$.

Relph E., 1976. Place and placelessness, London, Pion, 156 p.

Ruiz J., Domon G., 2013. Les « communautés de relations au paysage », l'expérience socio-spatiale avec le territoire comme nouveau cadre pour l'analyse des populations rurales, Géographie, économie, société, vol. 1, n 15, p. 139160.

Schmitz S. (dir.), 2012. Le «mode d'habiter » : analyse de l'usage d'une notion émergente en géographie, in O. LAZzarotti (dir.), Habiter. Vers un nouveau concept?, Paris, Armand Colin, p. 37-49.

SENCÉBÉ Y., 2011. Multi(ples) appartenances en milieu rural, Informations sociales, vol. 2, n 164 , p. 36-42.

Simard M., 2011. Transformation des campagnes et nouvelles populations rurales au Québec et en France : une introduction, Revue canadienne de développement régional, vol. 34, $\mathrm{n}^{\circ} 4$, p. 105-114.

Simard M., Guimond L., 2009. L'hétérogénéité des nouvelles populations rurales : comparaison dans deux MRC contras- 
tées au Québec, Recherches sociographiques, vol. L, n³ p. $475-505$.

Simard M., Guimond L., 2010. La migration de la ville vers la campagne au Québec? Portrait sociodémographique et économique de deux MRC contrastées et de leurs nouveaux résidents, Panorama des régions du Québec, édition 2010, Institut de la statistique du Québec, p. 13-29.

Simard M., Vézina J., Guimond L., [soumis pour publication]. Les néoruraux et leurs rapports aux décideurs locaux dans deux MRC au Québec : collaborations ou conflits?

Statistque Canada, 2011 . Recensement de 2011, Ottawa, Statistique Canada.
STock M., 2004. L'habiter comme pratique des lieux géographiques, EspacesTemps.net, Textuel, 18 déc. 2004. En ligne : [http://espacestemps.net/document 1 138.html].

Stockdale A., 2010. The diverse geographies of rural gentrification in Scotland, Journal of Rural Studies, vol. 26, p. 31-40.

Tuan Y.-F., 2005. [1977]. Space and Place. The Perspective of Experience, $4^{\text {th }}$ printing, Minneapolis, University of Minnesota Press, 248 p. 